and discuss three papers on each night of meeting, it were well to frame them of such a length as will admit of this. As this is a delicate subject for one to broach who is in momentary dread " ne me Crispini scrinia lippi compilasse putes," I shall, therefore, only so far allude to it as to bring to the recollection of my hearers the admirable advice of Horace upon this subject; taking the unpardonable liberty with this author and my audience of giving it as rendered by Francis, as it appears to me rather to have gained in point by the translation :-

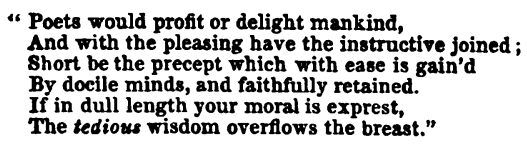

\section{HOSPITAL REPORTS.}

HEMTPHLEGA-DISEASE OF THE CARDIAC VALVES.-DEATH.

JoHn LYNCH, æt. 24, of sallow complexion and emaciated frame, was admitted into Guy's Hospital, March 22d, 1840, complaining of paralysis of the right side. He states that five months since he was suddenly seized with giddiness; that he then fell into a fit, and remained insensible for a fortnight; and that upon recovery from this state he found himself deprived of the use of the entire right side of his body. He has since been twice attacked with similar fits, but each of these lasted only about an hour. They were both attended with loss of voluntary power and sensation, and foaming at the mouth; but there was no struggling, neither did he, as far as he remembers, bite his tongue.

On admission, his articulation was imperfect, and the power of motion in the right upper and lower extremities very slight, if any; sensation, however, being perfect. There is a vacancy of expression in the countenance; the eyes are dull, and he suffers occasionally from vertigo. The heart's inpulse is very powerfully felt between the fourth and fifth ribs, and the second stroke is accompanied with a distinct bruit of a seesaw sound. On placing the hand over the region of the heart, a purring treinulous sensation is communicated to it. The pupils are naturally affected by light. Pulse 90 ; tongue coated; bowe!s regular; much thirst, but little desire for food. He seems to have lived temperately, and generally to have enjoyed good health previous to the first attack. The last fit occurred ten days since. To be cupped on the neck to eight ounces; blue pill, three grains, thrice a day; saline mixture, thrice a day.

On the 27th his mouth was becoming affected, and on the following day he was ordered to take only one pill daily.

April 1. Has not suffered from vertigo for several days. Pulse 100, compressible. The other symptoms remain much the same.

8. Speech improving, and he appears to be gradually regaining the use of the affected limbs. Pulse 105; tongue clean; no thirst; appetite good. He has not had any return of the vertigo.

10. He has had a fit this morning in his sleep; not ushered in by headaches, or any premonitory symptom. The patient in the next bed says that he saw him lying for five minutes apparently almost choked, and that he afterwards breathed heavily and foamed at the mouth. The fit lasted about twenty minutes, and he then fell asleep for seven hours. On waking he appeared to wander in his mind. The following morning he was ordered iodine, one grain; iodine of potass, twenty-four grains; tincture of lavender, four drachms; distilled water, six ounces.

15. There are occasionally involuntary twitchings about the arms and legs. He appears to be rather stronger in the affected side. There is no headache or giddiness. Heart's action still very powerful; the bruit heard at any
part of the chest.

20. He is stronger to-day than he has been for several days past. There is a constant pain in the right elbow, which he describes as similar to what he has felt in the shoulder when gaining strength in the arm. He has had a slight headache for half an hour after waking in the morning the last three days; and on lookirg downwards, or to either side, has had double vision. Heart's action not so violent; pulse 92 ; appetite good.

22: Has had another fit during the night. A few minutes after talking with the patient in the next bed, he was suddenly seized with trembling and insensibility, in which state he remained for half an hour; he then became violently convulsed - biting his tongue severely - for about ten minutes, and finally sank into a state (apparently) of coma. There were no immediate premonitory symptoms. He complains now (2 P.M.) of a slight headache. The cheeks are a little flushed. The pain in the elbow is gone off; heart's impulse less violent; pulse 92, soft; tongue clean; bowels open. He thinks the affected limbs are stronger. Ordered a seton in the neck.

29. Heart's impulse very great to day-sawing sound very distinct. Pulse 120, strong; no headache; tongue furred; bowels open.

May 3. There is double vision in left eye. When desirous of seeing clearly any distant object, he is obliged to close it and use the right eye only. Pupils obedient to light. No headache. Pulse 108, small, and compressible.

6. An abscess was opened to-day at the side of the neck. Pulse 100, jerking. He makes but little progress in regaining the use of the affected limbs. Has no headache. Other symptoms as before.

16. Got up to-day. Feels generally stronger. There is still the same vacant expression of countenance he has had all along. The heart's impulse is much less violent. Still has double vision on looking in certain directions. Pulse 108; tongue clean; appetite good.

21. Complains of pain in the forehead, not, he says, like that of common headache. Continues to see better when using the right eye only. Pupils contract and dilate naturally. No muscæ volitantes. Pulse 100, soft; bowels open twice daily. Ordered twelve leeches to the temples. 24. Weight of head and drowsiness relieved. Vision remains in the same state. Other symptoms as before. Three grains of sulphate of zinc, thrice a day.

June 1. His vision is much improved. There is no pain or weight about the head. The heart's impulse is by no means so violent as it was a fortnight since; and the thrilling sensation which was given to the hand is now quite absent. The bruit still exists. Pulse 92, not very compressible; tongue clean; appetite good. Continue remedies.

He continued much in the same state until the afternoon of the 20th, when he experienced considerable drowsiness and sense of weight about the head, with general indisposition. He therefore went to bed; and on the rurse going to see him at 12 P.M. she found him dead.

\section{Post-mortem Appearunces.}

On opening the chest, the upper lobe of the left lung was found to be adherent to the costal pleura; the right lung, however, being quite free. The structure of both was of a dark colour, being congested with blood, and odematous. The left ventricle of the heart, which was altogether enlarged, was dilated, and slightly hypertrophied. Only a small portion of the anterior attachment of the mitral valve remained, the rest having been destroyed by ulceration; but the posterior one was quite healthy. The lining membrane of the right auricle was thickened, and covered by a layer of firm lymph. The free margins of the sigmoid valves were considerably thickened, and partly destroyed by ulceration.

The membranes of the brain were found to be free from disease; but immediately below the surface of the left. hemisphere, in its posterior lobe, was found a cavity of the size of a pigeon's egg, and containing an old clot of extravasated blood, very firm, especially in its centre. The cortical and medullary structures immediately surrounding 
this appeared to have entirely lost their normal character. Each ventricle contained about half an ounce of serum. There was nothing unusual found in the rest of the brain.

The liver and kidneys were much congested with blood. In the spleen, which was more than twice its usual size, was found a clot of extravasated blood, exceedingly firm, and of a pale yellow colour. It was situated immediately below the surface of its convex margin. The mucous membrane of the colon was in several places destroyed by ulceration.

\section{NEWCASTLE-ON-TYNE, DURHAM, AND NORTHUMBERLAND INFIRMARY.}

\author{
PRACTICE OF MR. HEATH.
}

GANGRANa senilis, Followed by tetanus-death.

(Reported by Mr. Taylor, House Surgeon.)

Thos. Anderson, æt. 60, joiner, Winlaton, Newcastle, was admitted into this infirmary Nov. 12, 1840, under the care of Mr. Heath, for gangrene of the second toe of the left foot. He has a very florid complexion, dark hair; is of middling stuture, and has been very stout, but is now much emaciated; he has always been accustomed to a good deal of exercise, and, although in the habit of drinking spirits freely almost all his life, has always enjoyed good health prior to his present affection, with the exception of a slight rheumatic attack twenty years ago. He has suffered more or less pain in the affected toe for six months, which he attributes to having cut deep into a corn at that time; three months ago he suffered from vertigo, dimness of vision, œdema of the ankles, and scanty turbid urine, and was then much fatigued by sitting up with his wife, who was ill; he, however, paid little attention to these symptoms, and they subsided in a few days. About six weeks before he was admitted as a patient, the toe became red, swollen, and more painful, without any apparent disorder of his general health; in a little time the pain increased so much in severity as entirely to deprive him of rest; the toe assumed a livid appearance, and subsequently became quite black and devoid of sensation, presenting the following appearances on his admission:- 'The whole of the second toe, nearly up to the first joint, is quite black and devoid of sensation, with an oozing of serum from it; it has not lost any of its bulk; the foot itself is considerably swollen, and the integuments red and painful; the other toes are all sound; he suffers excruciating pain in the part, particularly at night; tongue clean ; bowels regular; appetite bad; no thirst; urine scanty and high coloured; skin soft and moist ; pulse 96, regular, but hard; respiration natural, lungs appear healthy on auscultation; heart's impulse somewhat increased, the systole accompanied by very slight bruit de souffet. Ordered 12 leeches to the foot; poultices, mild aperients, and three grains of opium every night.

14. He still suffers severely from pain, the opium having no effect in procuring rest ; the foot is still red and swollen; a line of demarcation is forming at the first joint of the toe; the integuments of the great toe have become inflamed, and there is a livid spot on the part adjacent to the diseased one. Leeches to be repeated, and poultices continued ; opium increased to four grains.

17. More pain and redness of foot; the dorsum of the great toe has become livid; pulse sharper and jerking; nights still passed in great suffering, although the dose of opium was doubled since last report, being eight grains every night. Leeches ordered to be applied again, and to take eighty drops of sedative solution of opium every night.

18. Has passed a worse night, and is altered in appearance ; countenance anxious, face more flushed, and is bedewed with cold, clammy perspiration; complains of stiffness of the lower jaw, which he is not able to open more than an inch ; no difficulty of deglutition; sense of constriction of chest; great thirst; no pain under sternum; severe pains shooting into the toes of the affected foot which has become paler, and the toe on each side of the gangrenous one has become livid.

19. The tetanic symptoms have increased much during the night, which he has passed in great suffering; complains of severe pain and stiffness in back of neck; the face is drawn to the left side by the spasmodic contraction of the sterno-cleido-mastoideus; he is much flushed and covered with profuse cold, clammy sweat; trismus more complete; deglutition very difficult ; features drawn into a risus sardonicus; the spasms affect the neck and back principally; occur frequently, but do not observe any regular periods; the spine forms an arch, the trunk being supported on the occiput and sacrum; intelligence good; speech unaffected; no difficulty of respiration; pain extending from sternum to spine not present; abdomen hard and incompressible; pulse 120, hard and jerking; the foot has become pale; the swelling has subsided; there is no extension of the gangrene, and he suffers no pain in the part; the spasms have become more frequent and severe; deglutition almost impossible, and he seems much weaker.

20. Has had no rest; the symptoms continue as before, but much aggravated; the spasins occur very frequently, in fact he is scarcely ever free from them; the face and chest are bathed in cold perspiration ; respiration difficult; collection of mucus in trachea; the pulse has lost its hardness and is increased in volume; intelligence perfect; the great toe has become gangrenous on its outer side; foot pale and free from pains; he continued in this state of suffering till 12 o'clock $A . x_{\text {., }}$ when he died.

The treatment, from the commencement of the tetanic symptoms, consisted in the administration of powerful purgatives of croton oil, \&c., turpentine enemata, opiates in large quantities, and calomel; but as no good effects were produced by these means, it is unnecessary to occupy room with details.

\section{ROYAL BERKSHIRE HOSPITAL.}

PRACTICE OF YR. BULLEY.

DISLOCATION OF THE FEMUR ON THE DORSUY ILIT.

Charles Mileer, aged 23, a stout muscular person working on the Great Western railway, was admitted into the hospital on August 21, on account of a dislocation of the right femur upon the dorsum ilii. On examination, it was found that the injured limb was shorter than the other by about two inches, incapable of extension; and, what is some what remarkable, without the slightest apparent inversion of the foot. There was an increased roundness of the joint, owing to the flat external surface of the trochanter major projecting directly outwards, giving it a more than usually bulged appearance, and differing in this respect from common cases, where the rotation inwards of the trochanter occasions the flattened appearance generally observed. The head of the bone was, from its peculiar position, (being placed, as it were, behind the trochanter, ) with difficulty felt upon the dorsum ilii, which difficulty was increased by the swelling which had taken place.

At first sight it seemed as if the bone had been fractured through the trochanter, but on rotating it outwards the head of the bone was felt to move in concert with the shaft, so that the signs of a dislocation were sufficiently well marked. The patient could himself flex the thigh upon the trunk to some extent; but the slightest attempt at abduction by another gave him very great pain.

The accident was occasioned by a quantity of falling earth having struck him on the back, as he was bending forwards, causing him to fall forcibly on the knee, which was at that moment crossed over the other. After bleeding to 12 ounces, and the use of the hot-bath, the reduction 